\title{
0xidative stress, hepcidin and nesfatin-I status in childhood iron and vitamin B12 deficiency anemias
}

\author{
Tünay K. Aşkar ${ }^{1, A-D}$, Olga Büyükleblebici², B, D, Adnan Adil Hismioğullar1 ${ }^{3, C, E, F}$, Zeynep Hünkerler, B \\ ${ }^{1}$ Department of Biochemistry, Health Sciences Faculty, Çankırı Karotekin University, Turkey \\ ${ }^{2}$ Department of Biochemistry, Veterinary Faculty, Aksaray University, Turkey \\ ${ }^{3}$ Department of Medical Biochemistry, Faculty of Medicine, Balıkesir University, Turkey \\ ${ }^{4}$ Department of Biochemistry, Çankırı Government Hospital, Turkey \\ A - research concept and design; $B$ - collection and/or assembly of data; C - data analysis and interpretation; \\ $D$ - writing the article; $E$ - critical revision of the article; $F$ - final approval of article
}

\section{Address for correspondence \\ Tünay K. Aşkar \\ E-mail: tunaykontas@yahoo.com}

Funding sources

None declared

\section{Conflict of interest}

None declared

Received on February 27, 2015

Revised on April 1, 2015

Accepted on April 25, 2016

DOI

10.17219/acem/ 62829

\section{Copyright}

\begin{abstract}
Background. Anemia is a disease that is long and often repetitive and can result in a great burden to the national economy. The most frequent nutritional deficiency anemias in children are related with iron and vitamin B12 deficiencies.
\end{abstract}

Objectives. The aim of this study was to determine the oxidative stress, hepcidin, and nesfatin-I levels in childhood iron and vitamin B12 deficiency anemias.

Material and methods. The study had 3 groups of 15 children, iron anemia deficiency group, vitamin B12 deficiency group and a control group.

Results. The TBARS and nesfatin-I levels were significantly higher in the iron and vitamin B12 deficiency groups and the total antioxidant levels were significantly lower when compared to the control group. In contrast, the plasma hepcidin levels were significantly lower in the iron deficiency group $(p<0.01)$ when compared to the control group; however, no significant differences were observed in the vitamin B12 deficiency group. Plasma homocysteine levels were significantly higher in the vitamin B12 deficiency group when compared to the control group ( $p<0.001$ ), but no differences were determined between the iron deficiency and control groups.

Conclusions. Our results showed that there are high levels of oxidative stress in childhood iron and vitamin B12 deficiency anemias, and we propose that plasma hepcidin and homocycteine levels may be useful in the differential diagnosis of childhood nutritional deficiency anemias. Nesfatin-1 hormone levels were identified for the first time in childhood iron deficiency and vitamin B12 deficiency anemias within this study and this hormone may also be useful in the differential diagnosis of anemias.

Key words: oxidative stress, hepcidin, nesfatin-I, childhood anemia 
Nutritional anemia is the most common blood related disorder in the world due to the inadequate intake of nutrients such us iron, folic acid and vitamin B12. According to the World Health Organization (WHO), 1/4 of the world's population has iron deficiency anemia. ${ }^{1}$ Iron, because of its ability to exchange electrons, plays an important role in oxygen transport, energy production, deoksiribonucleic acid (DNA), ribonucleic acid (RNA) and protein synthesis, which is why in iron deficiency anemia, hypochromic, microcytic anemia occurs. ${ }^{2}$ Although iron deficiency anemia is observed in all age groups, it is more prevalent in the 6-24 months age group and in adolescence it is recognized as the most common cause of anemia. ${ }^{3}$ It is diagnosed by measuring hemoglobin levels, in which a concentration less than $14 \mathrm{~g} / \mathrm{dL}$ in males and 12 $\mathrm{g} / \mathrm{dL}$ in females is considered diagnostic. ${ }^{4}$

Vitamin B12 is a water soluble vitamin synthesized mainly by microorganisms and its deficiency develops mainly due to inadequate intake of animal protein leading to megaloblastic anemia. ${ }^{5}$ The most important function of vitamin B12 is in the construction of DNA which is essential for the division and proliferation of cells and also for erythropoiesis. The physiological range of serum vitamin B12 is 200-900 pg/mL and levels below these display vitamin B12 deficiency symptoms. ${ }^{6}$

Hepcidin is a recently discovered hormone which is synthesized in the liver and is an antimicrobial protein displaying antibacterial and antifungal properties and maintains iron balance in the microorganism thus regulating its metabolism. When iron stores are adequate hepicidin production is increased in the liver and erythropoietic signals lead to the reduction in its synthesis. Hence, it can regulate the way iron is transported from enterocytes to the plasma in the small intestine. During anemia, a condition in which hypoxia and iron stores are low, hepcidin production is reduced; furthermore, hepcidin creates an important link between the body's defense, inflammation, and iron metabolism. ${ }^{7,8}$ A previous study has shown that during infection and inflammation, hepcidin synthesis is significantly increased due to stimulation by IL- $6 .{ }^{9}$

Nesfatin-1 is a satiety molecule that was first described in 2006. It is present in the hypothalamus and consists of 82 amino acids. ${ }^{10}$ It is found not only in the brain tissue but also in peripheral tissues such as the adipose tissue, stomach, pancreatic islets, liver, and the testis. Redundancy of nesfatin-1 causes loss of appetite and a decrease in body weight, In addition to this anorexigenic effect, it also promotes cardiac function, reduces the blood glucose level, and induces fear and anxiety-like behaviors; hence, it is a multifunctional peptide with anorectic effects. ${ }^{10}$

The increase in the amount of oxidative stress reactive oxygen species (ROS) is a condition characterized by a disorder of the antioxidant mechanisms. ${ }^{11}$ Free oxygen radicals are being deactivated by the antioxidant system in the organism and a balance between oxidative stress and antioxidant systems is maintained; if this balance is disturbed, tissue damage may occur. Toxicity that is generated by free oxygen radicals increases in the presence of transition metals such as iron or copper. ${ }^{12}$ It has been demonstrated that people who are iron deficient have abnormalities, in erythrocytes, and in protective mechanisms against oxidative damage. On the other hand, an increase in iron deposition creates hydroxyl radicals with Fenton and Haber-Weiss reactions, leading to DNA damage and cytotoxicity. ${ }^{13}$

The possible impact of oxidative damage due to iron and vitamin B12 deficiency in children and the resulting damage to the children's physical and mental development has not yet been fully clarified. The determination of oxidative damage due to childhood anemias can possibly make a significant contribution to the diagnosis and treatment of this disease.

For this reason, this study was conducted in order to determine the oxidative stress levels, hepcidin, and nesfatin-I hormone levels in childhood iron and vitamin B12 deficiency anemias. For the determination of oxidative stress, thiobarbituric acid derivatives (TBARS), and total antioxidant capacity (TAC) levels were determined.

\section{Material and methods}

This study was approved by the Ethics Committee from Bülent Ecevit University School of Medicine and was conducted on a group of children aged 4-9 years (average mean age $6.1 \pm 1.4$ years) that came to the Çankırı State Hospital Pediatric Outpatient Clinic between September 2011 and May 2012. Fifteen children were assigned to each of the 3 groups, iron deficiency anemia, vitamin B12 deficiency anemia, and an apparently healthy group (control group). The exclusion criteria included children suffering from inflammatory diseases, children with a known chronic disease and who had taken antibiotics in the last 4 weeks. Iron deficiency anemia group was composed of children who had hemoglobin $(\mathrm{Hb})$ level of $<11.5 \mathrm{~g} / \mathrm{dL}$, serum iron $<30 \mathrm{mg} / \mathrm{dL}$ and ferritin $<15 \mathrm{ng} / \mathrm{mL}$ and, vitamin B12 deficiency group had $\mathrm{Hb}$ level of $<11.5 \mathrm{~g} / \mathrm{dL}$, and vitamin B12 levels of $<200 \mathrm{pg} / \mathrm{dL}$.

Three $\mathrm{mL}$ of venous blood was taken from the children and placed in tubes containing anticoagulant and the plasma was separated for biochemical analysis. In order to inhibit fragmentizing of nesfatin-1 hormone by serine protease $2 \mathrm{mg} / \mathrm{mL}$ 4-[2-aminoethyl benzene] sulfonyl fluoride (AEBSF) was added to anticoagulant tubes. Plasma samples were placed into the eppendorf tube and stored at $-80^{\circ} \mathrm{C}$, until analyses.

\section{Laboratory methods}

Plasma iron, ferritin, hemoglobin and vitamin B12 levels were performed on an automated analyzer (Roche, DP Moduler System, Tokyo, Japan) using commercial test 
kits. For the quantitative measurement of TBARS, Oxiselect $^{\mathrm{TM}}$ TBARS Assay Kit (Cell Biolabs, USA) were used. The Oxiselect ${ }^{\mathrm{TM}}$ TBARS Assay Kit is a tool for the direct quantitative measurement of malondialdehyde (MDA) in biological samples. Plasma TAC levels were measured by using Rel Assay Diagnostics kit (Mega Tip, Turkey). This method was based on bleaching the distinct color of the 2.2'-azino-bis (3-ethylbenzothiazoline-6-sulfonic acid) radical cation via the effects of the antioxidants.

Plasma homocysteine levels were determined using Axis (IBL, Germany) homocysteine enzyme-immunoassay kit. Hepcidin (DRG International Inc., USA) and Nesfatin-I hormone levels were measured by enzyme linked immunosorbent assay (ELISA) using a commercially available kit (Phoenix Pharmaceuticals Inc., USA). The procedures for determining the hormone levels were performed as recommended in the relevant catalogues, using micropleyt reader ( $\mu$ Quant Elisa reader, Bio-Tek, USA).

\section{Statistical analysis}

The data obtained in this study was evaluated using a statistical computer package (SPSS 13 for Windows standard version). Data averages were expressed as \pm standard error. The normality tests of the data was subjected to statistical differences between groups, by a one- way analysis of variance (ANOVA), and the Duncan test as a post-test was applied.

\section{Results}

Serum iron (Fe), hemoglobin $(\mathrm{Hb})$, ferritin and B12 values of the iron deficiency group, vitamin B12 deficiency group and control are presented in Table 1. In the study, while iron, hemoglobin, and ferritin levels were significantly lower $(\mathrm{p}<0.001)$ in the iron deficiency group, in vitamin B12 deficiency group hemoglobin and vitamin B12 levels were significantly lower when compared to the control group ( $\mathrm{p}<0.01, \mathrm{p}<0.001)$ respectively.

The TBARS and nesfatin-I levels were significantly higher in the iron and vitamin B12 deficiency groups and the total antioxidant levels were significantly lower when compared to the control group ( $\mathrm{p}<0.001$, $\mathrm{p}<0.01)$ respectively.

In contrast, the serum hepcidin levels were significantly lower in the iron deficiency group $(\mathrm{p}<0.01)$ compared to the control; however, no significant differences were observed when vitamin B12 deficiency group was compared to the control group. Serum homocysteine levels were significantly higher in the vitamin B12 deficiency group when compared to the control group ( $p<0.001)$, but no differences were apparent between the iron defi-

Table 1. Iron and vitamin B12 status parameters in anemia and control groups

\begin{tabular}{|c|c|c|c|}
\hline Parameters & Control group & Iron deficiency anemia & Vitamin B12 deficiency anemia \\
\hline $\mathrm{Fe}(\mu \mathrm{g} / \mathrm{dL})$ & $55.1 \pm 20.5$ & $26.2 \pm 17.3^{*}$ & $60.8 \pm 23.9$ \\
\hline $\mathrm{Hb}(\mathrm{g} / \mathrm{dL})$ & $13.8 \pm 3.4$ & $7.9 \pm 4.2^{*}$ & $10.1 \pm 2.7$ \\
\hline Ferritin (ng/mL) & $34.7 \pm 12.3$ & $11.3 \pm 4.7^{*}$ & $35.9 \pm 15.4$ \\
\hline Vitamin B12 (pg/dL) & $281 \pm 101$ & $265 \pm 94$ & $148.1 \pm 87^{*}$ \\
\hline
\end{tabular}

*signs indicate statistical differences among the groups ( $p<0.05)$. Fe; iron, Hb; hemoglobin.

Table 2. Oxidative stress, hepcidin, and nesfatin-I status in anemia and control groups

\begin{tabular}{|c|c|c|c|}
\hline Parameters & Control group & Iron deficiency anemia & Vitamin B12 deficiency anemia \\
\hline TBARS (nmol/L) & $2.01 \pm 0.6$ & $2.97 \pm 0.5^{*}$ & $3.14 \pm 0.8^{*}$ \\
\hline TAC (mmol/L) & $1.35 \pm 0.4$ & $0.76 \pm 0.2^{*}$ & $0.84 \pm 0.3^{*}$ \\
\hline Homocycteine ( $\mu \mathrm{mol} / \mathrm{L})$ & $10.3 \pm 6.2$ & $11.7 \pm 5.6$ & $33.1 \pm 9.7^{*}$ \\
\hline Hepcidin (ng/mL) & $206.2 \pm 41$ & $163.7 \pm 64^{*}$ & $218.5 \pm 43$ \\
\hline Nesfatin-I (ng/mL) & $1.81 \pm 1.0$ & $3.63 \pm 1.5^{*}$ & $3.51 \pm 1.3^{*}$ \\
\hline
\end{tabular}

* signs indicate statistical differences among the groups $(\mathrm{p}<0.05)$. TBARS - thiobarbituric acid derivatives; TAC - total antioxidant capacity. 
ciency group and the control group. TBARS, TAC, hepcidin, nesfatin-I and homocysteine levels of children who have childhood iron deficiency anemia and vitamin B12 deficiency are given in Table 2.

\section{Discussion}

Iron deficiency anemia and vitamin B12 deficiency is largely associated with people of low socio-economic background and people living in less developed countries. It is also prevalent in developed countries due to nutritional deficiencies or disorders, and is the most common type of anemia diagnosed. ${ }^{14}$

During the metabolic and physiological processes in the body, large numbers of detrimental reactive oxygen species are produced and may lead to pathological reactions due to oxidative reactions. These free radicals are neutralized within the body by enzymatic or non-enzymatic antioxidant mechanisms. Reactive oxygen species has been shown to damage the membrane of the erythrocytes, resulting in the alteration of their structure. ${ }^{15}$

In this study, children with iron deficiency anemia and vitamin B12 deficiency anemia displayed significantly higher oxidative stress levels as measured by TBARS levels compared to the control group ( $p<0.001$ ), and the total antioxidant capacity levels were found to be significantly lower than those in the control group ( $\mathrm{p}<0.01$ ). In agreement with our study, their study in patients with iron deficiency anemia also reported an increase in the levels of oxidant products, such as malondialdehyde and a decrease in the levels of the antioxidant enzymes, such as glutathione peroxidase. ${ }^{16}$ In anemias, due to Thalassemia and G6PD enzyme deficiency, it has been shown that oxidative stress increases and antioxidant capacity is reduced. ${ }^{17}$ In contrast, Acharya et al. reported an increase in the levels of antioxidant enzymes in patients with iron deficiency anemia, and they suggested that this was due to overcompensation against the induced oxidative stress. ${ }^{18}$ Al-Maskari et al. reported that hyperhomocysteinemia with folate and vitamin B12 deficiency causes oxidative stress and there is also a parallel decline in the total antioxidant capacity. ${ }^{19}$ In the study reported here, high levels of TBARS present in vitamin B12 deficiency anemia and the levels of homocysteine in patients with high and low levels of TBARS and TAC are in agreement with Al-Maskari et al. ${ }^{19}$ In this study, high TBARS identified in B12 deficiency anemia groups and homocysteine levels is compatible with the observed low TAC levels.

Oxidative stress is induced as a result of the elevation of homocysteine, which in plasma is oxidized rapidly to disulfide homocysteine. During this reaction, reactive oxygen species (ROS) are formed as hydrogen peroxide radical. ${ }^{20}$ Homocysteine metabolism is associated with metabolism B12, and vitamin B12 deficiency leads to an increase in homocysteine levels and thus to an increase in amount of ROS derivatives. Therefore, in our study, in children with vitamin B12 deficiency, it was found that serum homocysteine levels were significantly higher $(\mathrm{p}<0.001)$ than those in the control group; when levels in children with iron deficiency anemia were compared with the control group, there was no statistically significant change. This is in agreement with Savage et al., who in their study of 406 people, also reported high levels of homocysteine in vitamin B12 deficiency anemia patients. ${ }^{21}$

Hepcidin is a peptide hormone synthesized from the liver. It is removed from the blood and excreted in the urine, and is a major regulator of systemic iron balance. ${ }^{22}$ Ulukol et al., in a study conducted on 16 children with anemia and 54 without anemia, have shown that serum hepcidin levels in patients with anemia are lower than those in the non-anemic group. ${ }^{23}$ Also, in the study reported here, hepcidin levels were significantly lower in the group with iron deficiency anemia compared to the control group, which seems to support the study of Ulukol et al. In the conducted study in children with vitamin B12 deficiency anemia, serum hepcidin levels were similar to those of the control group.

Nesfatin-1 is a peptide hormone that is produced in the brain of mammals and is responsible for the production of body fat and the regulation of appetite. The studied conducted have shown that excess nesfatin-1 in the brain leads to a loss of appetite, less frequent hunger, and a drop in body fat and weigh. ${ }^{10}$ Abacl et al. determined that serum nesfatin-1 values in obese children are also decreased. ${ }^{24}$ In iron and vitamin B12 deficiency anemia, symptoms such as loss of appetite, fatigue and weakness are observed. Therefore, in our study, the presence of nesfatin-1 levels were significantly higher in the anemia groups when compared to the control group $(\mathrm{p}<0.001)$ and may be associated with the loss of appetite that is observed in anemias, resulting in fatigue, and growth retardation.

Our results showed that oxidative stress occurs in children with iron deficiency and vitamin B12 deficiency anemias has been identified with high TBARS levels and low total antioxidant capacity levels. And we propose that measuring plasma hepcidin and homocycteine levels may be useful in the differential diagnosis of childhood nutritional deficiency anemias. And nesfatin-1 hormone levels were identified for the first time in childhood iron deficiency and vitamin B12 deficiency anemias within this study and this hormone may be responsible for the symptoms of the disease.

But there is insufficient data in the literature about the roles of hormone hepcidin, which is an antimicrobial hormone, and nesfatin-I in the pathophysiology of anemias. Therefore, there is a need for further studies to determine the relationship between the nesfatin-I and hepcidin hormones and the mechanism of weight loss in childhood anemias. 


\section{References}

1. Dallman PR. Progress in the prevention of iron deficiency in infants. Acta Pediatr. 1990;365:2837.

2. Buratti P, Gammella E, Rybinska I, Cairo G, Recalcati S. Recent advances in iron metabolism: Relevance for health, exercise, and performance. Med Sci Sports Exerc. 2014;9. doi: 10.1249/ MSS.0000000000000593.

3. Brugnara C. Iron deficiency and erythropoiesis. New diagnostic approaches. Clin Chemistry. 2003;4:1573-1578.

4. Thomas C, Thomas L. Biochemical markers and hematologic indices in the diagnosis of functional iron deficiency. Clin Chem. 2002;4:1066-1076.

5. Bjørke-Monsen AL, Ueland PM. Cobalamin status in children. J Inherit Metab Dis. 2011;34(1):111-119.

6. Altay C, Cetin M. Vitamin B12 absorption test and oral treatment in 14 children with selective vitamin B12 malabsorption. Pediatr Hematol Oncol. 1999;16(2):159-163.

7. Ganz T. Hepcidin: A regulator of intestinal iron absorption and iron recycling by macrophages. Best Practice \& Research Clinical Haematology. 2005;18(2):171-182.

8. Rossi E. Hepcidin: The iron regulatory hormone. Clin Biochem Rev. 2005;26(3):47-49.

9. Ganz T. Hepcidin: A key regulator of iron metabolism and mediator of anemia of inflammation. Blood. 2003;102(3):783-788.

10. Stengel A, Mori $M$, Taché $Y$. The role of nesfatin- 1 in the regulation of food intake and body weight: recent developments and future endeavors. Obes Rev. 2013;14(11):859-870.

11. Inal M, Akyüz F, Turgut A, Getsfrid WM. Effect of aerobic andaAnaerobic metabolism on free radical generation swimmers. Med Sci Sports Exerc. 2001;33:564-567.

12. Özkan A, Fışkın K. Serbest oksijen radikalleri ve Karsinogenez ve antioksidan enzimler. International Journal of Hematology and Oncology. 2004;14(1):52-60.
13. Gutteridge JM. Biological origin of free radicals, and mechanisms of antioxidant protection. Chem Biol Interact. 1994;91(2-3):133-140.

14. Domellöf $M$. Iron requirements, absorption and metabolism in infancy and childhood. Curr Opin Clin Nutr Metab Care. 2007;10(3):329-335.

15. Mccord JM. Human disease, free radicals and the oxidant/antioxidant balance. Clin Biochem. 1993;26:351-357.

16. Kurtoglu E, Ugur A, Baltaci AK, Undar L. Effect of iron supplementation on oxidative stress and antioxidant status in iron-deficiency anemia. Biol Trace Elem Res. 2003;96:117-123.

17. Cheng ML, Ho HY, Tseng HC, Lee CH, Shih LY, Chiu DT. Antioxidant deficit and enhanced susceptibility to oxidative damage in individuals with different forms of alpha-thalassaemia. Br J Haematol. 2005;128:119-127.

18. Acharya J, Punchard NA, Taylor JA, Thompson RP, Pearson TC. Red cell lipid peroxidation and antioxidant enzymes in iron deficiency. Eur J Haematol. 1991;47:287-291.

19. Al-Maskari MY, Waly MI, Ali A, Al-Shuaibi YS, Ouhtit A. Folate and vitamin B12 deficiency and hyperhomocysteinemia promote oxidative stress in adult type 2 diabetes. Nutrition. 2012;28:23-26.

20. Diaz-Arrastia, R. Homocycteine and neurologic disease. Arch Neurol. 2000;7:1422-1428.

21. Savage DG, Lindenbaum J, Stabler SP. Sensitivity of serum methylmalonic acid and total homocysteine derterminations for diagnosing cobalamin and folate deficiencies. Am J Med. 1994;96:239-246.

22. Anderson GJ, Darshan D, Wilkins SJ, Frazer DM. Regulation of systemic iron homeostasis: How the body responds to changes in iron demand. Biometals. 2007;20:665-674.

23. Ulukol B, Orhon FS, Hanoluk A, Akar N. Serum pro-hepcidin levels in infants with iron deficiency anaemia. Int JnI Lab Hem. 2008;30:546-547.

24. Abacı A, Catlı G, Anık A, Kume T, Bober E. The relation of serum nesfatin-1 level with metabolic and clinical parameters in obese and healthy children. Pediatr Diabetes. 2013;14:189-195. 
